\title{
An NMR study on internal browning in pears
}

\author{
N. Hernández-Sánchez ，B.P. Hills ，P. Barreiro , N. Marigheto \\ Physical Properties Laboratory-Advanced Technologies in Agrifood, E.T.S.I. Agronomos, Polytechnic University of Madrid, \\ Avda. Complutense s/n, 28040 Madrid, Spain \\ Institute of Food Research. Norwich Reseurch Park. Colney. Norwich NR4 7UA, United Kingdom
}

\begin{abstract}
lnternal browning in pears (Pyrus communis L. cv. Blanquilla) has been studied by NMR and MRl in order to develop a non-destructive procedure for on-line disorder identification. For NMR relaxometry, disordered tissue shows higher transverse relaxation rates compared to sound tissue, especially at higher magnetic field strength and for long pulse spacing. Membrane alteration and therefore tissue disintegration, as well as water evaporation, appear to be the main causes of this response. Correlation between relaxation times and diffusion showed that the proton pools in disordered lissue are grouped into a smaller number of populations compared to sound tissue, also highlighting cell decompartmentation in disordered tissue. At a macroscopic level, fast low angle shot MR images, effective transverse relaxation-weighted (TR $11 \mathrm{~ms}$ and TE $3.7 \mathrm{~ms}$ ) and proton density-weighted (TR $7.6 \mathrm{~ms}$ and TE $2.5 \mathrm{~ms}$ ), were acquired for pears at a rate of $54 \mathrm{~mm} / \mathrm{s}$. Images have been discriminated for internal breakdown according to histogram characteristics. Up to 94 and $96 \%$ of pears, respectively, were correctly classified in the former and the latter type of images. In this study a minimum value of $12 \%$ of tissue affected by breakdown was always clearly identified.
\end{abstract}

Keywords: Internal browning; Pear; Fruit quality; Physiological disorder; On-line MRI; Image processing

\section{Introduction}

Internal browning in pears is characterized by softening and browning of tissues and development of cavities. It is an important postharvest disorder that is observable only at the end of the commercial chain, since the external appearance of the fruit is not altered, even when the characteristic brown coloured tissue is widely spread from the core to the surrounding flesh. There have been many studies on the factors influencing the development of this disorder and gas composition during controlled atmosphere (CA) storage has been identified as the primary influence. Various names have been applied to the disorder since it has been difficult to establish clear differences on the basis of similar symptoms. Among these are internal breakdown, brown heart, brown core, and core breakdown.

Kader (1989) attributed the flesh browning in 'Bartlett' pears to $\mathrm{CO}_{2}$ injury (among other causes such as bruising) and related such coloration to the degradation of phenols into quinones by means of polyphenol oxidase (PPO), the quinones subse- quently polymerizing into brown-coloured pigments. Veltman et al. (2000) found that the concentration of an antioxidant, ascorbic acid (AA), was reduced in 'Conference' and 'Rocha' pears by browning-inducing conditions such as enhanced $\mathrm{CO}_{2}$ and lowered $\mathrm{O}_{2}$. Veltman et al. (2003) carried out a study to assess the relation between the internal browning in 'Conference' pears and the decrease in antioxidant levels as well as in energy availability. Under hypoxia there is limited $\mathrm{O}_{2}$ availability for respiration and cells may be unable to generate the necessary energy for metabolism. Results suggest that membrane maintenance and free radical control by means of AA, glutathione and $\alpha$-tocopherol are altered, leading to cell decompartmentation and browning reactions. The importance of energy status in brown heart development in 'Conference' pears has been demonstrated by Saquet et al. (2003). High ATP concentration in the fruit tissue and energy charge was associated with a reduction in the disorder incidence.

Lammertyn et al. (2000) performed a multivariate analysis to establish prediction and classification models for internal browning. High $\mathrm{CO}_{2}$ and low $\mathrm{O}_{2}$ concentrations along with longer storage time, over maturity, heavy fruit and hard tissue were characteristics favouring the development of the internal disorder. The developed model correctly classified up to $88 \%$ of the 
Table 1

Summary of the experiments performed on "Blanquilla" pears with different levels of damage

\begin{tabular}{|c|c|c|}
\hline & Samples & NMR eguipment \\
\hline \multicolumn{3}{|l|}{ IFR experiments } \\
\hline \multicolumn{3}{|l|}{ NMR relaxonetry } \\
\hline C.PMG sequences with variable $90-180^{\circ}$ pulse spacing & 24 tissue samples & $\begin{array}{l}\text { Resonance Instruments DRX operating at } 100.13 \text { and } \\
300.15 \mathrm{MHz}\end{array}$ \\
\hline$T_{1}-T_{2}$ correlation spectroscopy & 20 tissue samples & $\begin{array}{l}\text { Resonance Instruments DRX operating at } 23.4,100.13 \\
\text { and } 300.15 \mathrm{MHz}\end{array}$ \\
\hline$T_{2}-D$ cortelation spectroscop & 6 tissue samples & Resonance Instruments DRX operating at $23.4 \mathrm{MHz}$ \\
\hline \multicolumn{3}{|l|}{ LPF-UCM expetiments } \\
\hline \multicolumn{3}{|l|}{ NMR relaxometry } \\
\hline$T_{2}$ maps & 3 stationary pears & $\begin{array}{l}\text { Bruker Biospec spectrometer operating at } 200 \mathrm{MHz} \\
(20 \mathrm{~cm} \text { available diameter) }\end{array}$ \\
\hline \multicolumn{3}{|l|}{ MRI } \\
\hline $\begin{array}{l}\text { FLASH } T_{2}^{*} \text {-weighted images (longitudinal tomography) } \\
\text { FLASH } T_{2}^{*} \text { and DP-weighted images (ttansvetsal tomography) }\end{array}$ & $\begin{array}{l}4 \text { stationary pears } \\
32 \text { peats in motion }\end{array}$ & $\begin{array}{l}\text { Bruket Biospec spectrometer operating at } 200 \mathrm{MHz} \\
(20 \mathrm{~cm} \text { available diameter) }\end{array}$ \\
\hline
\end{tabular}

IFR stands for Institute of Food Research, United Kingdom; LPF stands for Physical Properties Laboratory-Advanced Technologies in Agrifood, Spain; and UCM stands for Complutense University of Madrid. Spain.

pears. These authors also confirmed that the cavities appearing in disordered pears grow at the expense of the brown tissue.

Larrigaudière et al. (2004) also used a multivariate analysis to clarify whether core browning and brown heart are the product of a single disorder or are two different ones. The analysis showed that both disorders may not be clearly differentiated, and that both begin with a fermentative process induced by high concentration of $\mathrm{CO}_{2}$ and energy charge reduction. These authors suggested that for core browning, the tissue breakdown is related to senescence accelerated by high $\mathrm{CO}_{2}$ concentrations and is caused by the accumulation of fermentative products such as acetaldehyde and ethanol, which are toxic for the cells. In contrast, brown heart results from a decrease in antioxidant synthesis and/or regeneration with subsequent tissue alteration by oxidation reactions.

The results of Larrigaudière et al. (2004) show that when pears are exposed to either disorder-inducing conditions, the cell membranes lose their integrity, which results in changes in water compartmentation. Such variation, along with the lack of external symptoms, suggests that non-destructive NMR and MRI are appropriate for internal inspection after storage.

Several authors have used these techniques to study core breakdown development in 'Bartlett' and 'Conference' pears. Wang and Wang (1989) detected an increase in free water in 'Bartlett' pears as high intensity areas and the occurrence of air spaces as dark areas in the disordered region in $T_{1}$-weighted images. Lammertyn et al. (2003a,b) studied the spatial distribution as well as the time course of the disorder by means of MRI and X-ray computer tomography. A constant pixel intensity threshold was set to separate and quantify disordered and sound tissue within the images. The contrast between sound and brown tissue was followed over time. MRI offered a higher sensitivity for detecting incipient browning and a better contrast between disordered and sound tissues than X-ray. This showed that the disorder does not grow spatially over time but only increases in contrast magnitude. These earlier NMR studies were undertaken on stationary fruit and with slow imaging sequences unsuitable for rapid on-line detection of the internal disorders.
In this paper we extend available studies using a variety of exploratory NMR sequences on 'Blanquilla' pears. These NMR studies are complemented with optical microscopy.

\section{Materials and methods}

This study was been carried out within a joint research programme between the Physical Properties Laboratory-Advanced Technologies in Agrifood (LPF-TAG) and the Institute of Food Research (IFR). All the samples have been prepared by LPFTAG. The microscopic study has been performed at the NMR facilities at IFR, while the macroscopic study took place at Complutense University of Madrid tacilities. The experimental set up is summarized in Table 1 in terms of exploratory techniques, samples and NMR equipment.

\subsection{Samples}

Pears (Pynus communis L. cv. Blanquilla) were obtained from the central fruit market in Madrid. Fruit were sorted by an expert in fruit handling into potentially non-disordered and disordered samples according to the information on storage length provided by wholesalers. The former fruit were directly stored at $1{ }^{\circ} \mathrm{C}$, whereas the later were first sealed in containers to increase the $\mathrm{CO}_{2}$ concentration before being stored at $1^{\circ} \mathrm{C}$. For macroscopic NMR, $T_{2}$ maps and static MR images were obtained from whole pears with potential different levels of damage, including no disorder. Temperature stabilisation was the only requirement for preparation of such samples.

For microscopic NMR studies, pears were transversally cut in half and then radial rectangular prisms and cylinders were extracted from both the core and the flesh tissue. Pieces of tissue were placed into sampling tubes, sealed to avoid dehydration, and the temperature stabilised at $26^{\circ} \mathrm{C}$ before exploratory measurements.

For MRI on-line testing, whole fruit were placed on a dedicated conveyor belt (Hernández-Sánchez et al., 2004) working at 
$54 \mathrm{~mm} / \mathrm{s}$ after reaching room temperature $\left(22^{\circ} \mathrm{C}\right.$, no temperature control was performed during image acquisiton).

\subsection{NMR relaxometry}

The relaxation measurements performed at IFR included: (a) Carr-Purcell-Meiboom-Gill (CPMG) sequence with variable 90-180 pulse spacing ( $\tau$ ); (b) $T_{2}$ maps; (c) $T_{1}-T_{2}$ correlation spectroscopy; (d) $T_{2}$-diffusion correlation spectroscopy ( $T_{2}-D$ ). Resonance Instruments DRX spectrometers operating at 23.4, 100.13 and $300.15 \mathrm{MHz}$ were employed for (a)-(d), whereas a Bruker Biospec spectrometer operating at $200 \mathrm{MHz}$ was used for (b).

Transverse relaxation time distributions were measured using the Carr-Purcell-Meiboom-Gill (CPMG) sequence consisting of a $90^{\circ}$ pulse followed by an echo train induced by successive $180^{\circ}$ pulses. The $90-180^{\circ}$ pulse spacing $(\tau)$ was varied between 200 and $10,000 \mu s$. The echo decay envelope was inverse Laplace transformed with Resonance Instruments WINDXP software to compute the $T_{2}$ value of the main proton pool to try to assess whether the $\tau$ effect on the computed $T_{2}$ values was different for fresh and disordered tissue.

To obtain the probability density of different proton pools distinguished on the basis of their $T_{1}$ and $T_{2}$ values, twodimensional ( $T_{1}-T_{2}$ ) cross-correlation spectra were acquired by setting $\tau$ to 200 and $4000 \mu$ s and performing $2 \mathrm{D}$-inverse Laplace transformation (Hills et al., 2004).

Proton pool assignment for tissue characterization was implemented by performing $\left(T_{2}-D\right)$ correlation spectroscopy (Marigheto et al., 2005). The $T_{2}$ of the different water subcellular compartments are associated with water diffusion constants, $D$ $\left(\mathrm{m}^{2} / \mathrm{s}\right)$. Experiments were performed with a combined sequence (CPMG-PGSE) in which the CPMG is followed by the pulsed gradient spin-eco sequence (Stejskal and Tanner, 1965). The number of $180^{\circ}$ pulses, $n$, varied between 2 and 750 and for each $n$ setting the echo amplitude was measured at diftusion gradient values, $G$, ranging between 0 and $1.175 \mathrm{~T} \mathrm{~m}^{-1} .\left(T_{2}-D\right)$ correlation spectroscopy also allowed evaluation of the effect of the internal browning on the water diffusivity of the cell compartments.

The NMR relaxometry performed at (LPF-UCM) included $T_{2}$ maps of $5 \mathrm{~mm}$ thickness slices and $12 \mathrm{~cm}$ field of view (FOV) obtained by imaging pears as $256 \times 256$ matrices with the standard two-dimensional (2D) spin echo sequence. Thirty echoes were collected with a $3472 \mathrm{~ms}$ repetition time (TR) and $16 \mathrm{~ms}$ echo time (TE). The magnitude of these multiecho images was fitted on a pixel-by-pixel basis with a two-parameter ( $T_{2}$ and the signal intensity at the equilibrium) monoexponential function without baseline, using the standard Bruker imaging Fit Package.

\subsection{On-line MRI acquisition}

A Bruker Biospec spectrometer operating at $200 \mathrm{MHz}$ was used for dynamic experiments. The bore of the magnet is horizontal with a $40 \mathrm{~cm}$ diameter reduced to $26 \mathrm{~cm}$ when the gradients stack in place. A birdcage coil with an inner diameter of $20 \mathrm{~cm}$ was used. The on-line conditions were simulated with a prototype conveyor system (Hernández-Sánchez et al,, 2004) exclusively designed to operate under the magnetic working conditions. The design was based on that developed by Chen et al. (1996).

Potentially disordered pears were imaged at LPF-UCM under static conditions in order to address the regions presenting higher probability for disorder onset allowing a proper selection of fruit orientation, which would be used for further on-line measurements. The acquisition of MR images for conveyed samples requires fast sequences which minimize the motion effect on image quality and reduce the acquisition time below $1 \mathrm{~s}$ (premise for realistic on-line applications). Therefore, inspection at LPFUCM was developed by means of a fast low angle shot (FLASH) setting the image sequence parameters as to enhance tissue contrast and facilitating disorder identification: TR of $11.14 \mathrm{~ms}$, TE of $3.9 \mathrm{~ms}$, flip angle of $10^{\circ}$. Three images per sample were obtained from longitudinal slices. The FOV was set to $12 \mathrm{~cm}$, and the slice thickness to $10 \mathrm{~mm}$, the slice-to-slice distance used was $10 \mathrm{~mm}$ being the matrix acquisition size $256 \times 256$.

For on-line inspection (three repetitions per sample), coronal images (horizontal field of view lying on plane $x z$ ) were acquired from transverse slices at the equatorial zone of the fruit as this location was identified as the best one for disorder inspection. Pears were placed in the conveyor belt with the stem-calyx axis along the $y$-direction (vertical direction). Two types of FLASH pulse sequence were used. One was for effective transverse relaxation time $\left(T_{2}^{*}\right)$ weighted images, with TR $11 \mathrm{~ms}$ and TE $3.7 \mathrm{~ms}$. Such time constant characterizes relaxation processes where the applied magnetic field is not perfectly homogeneous. The other sequence was for proton density (PD) weighted images with TR $7.6 \mathrm{~ms}$ and TE $2.5 \mathrm{~ms}$. For both types of images the flip angle was set to $10^{\circ}$ with $12 \mathrm{~cm}$ FOV and $10 \mathrm{~mm}$ slice thickness. Pulse gains, attenuator, shimming settings as well as FOV location were adjusted for a sound sample with average dimensions and were then held constant for all samples. The acquisition matrix size was $64 \times 128$, with a total acquisition time of $703 \mathrm{~ms}$ and 484 for $T_{2}^{*}$-weighted and PD-weighted images, respectively. Images of fruit conveyed at $54 \mathrm{~mm} / \mathrm{s}$ were collected.

To reconstruct the images a dedicated algorithm was used consisting of an automated correction of the baseline in the $k$-space lines as well as of the phase-shift caused by the sample motion during signal acquisition (Hernández et al,, 2005). Fourier shift theorem was applied and the dephasing generated in the acquired echo signal was corrected through the computation of a phase factor dependent on sample displacement. Zero-filling to $128 \times 128$ matrix size during Fourier transformation was also applied in order to improve spatial resolution.

\subsection{Image processing and data analysis}

\subsubsection{Automated image features extraction}

MR images were corrected for motion induced artifacts by applying a dedicated algorithm (Hernández et al, 2005) based on the Fourier shift theorem (Bracewell, 2000). Then, motion corrected images were processed to extract a number of 
texture features from the region of interest (ROI), in our case this region included both flesh and core tissues of the fruit. A devoted algorithm for automated ROI segmentation on oranges (Hernández-Sánchez et al., 2004) was applied and adjusted to the current application on pears. In order to characterize the image texture the signal intensity histogram (256 categories) of those pixels within the ROI was obtained. Then, the following features were extracted: coefficient of variation for signal intensity (SCV), skewness, kurtosis, percentage of accumulated pixels for selected histogram categories C3 (accumulated pixels up to 3 rd category), C26 (accumulated pixels up to 26th category), C64 (accumulated pixels up to 64th category) and C128 (accumulated pixels up to 128th category); and number of pixels left after accumulation for C192, C230 and C253 categories. Those parameters that enabled disorder identification by means of ANOVA analysis were selected for further study.

\subsubsection{Data analysis}

As indicated, analysis of variance was performed with software package STATISTICA (StatSoft, Inc.) to address most significant features according to differences between sound and disordered pears (classification made by visual inspection after cutting in halves). A threshold was obtained from each feature as extracted from a logistic regression at $90 \%$ probability of fruit not being disordered. As well, significant features were introduced into a forward stepwise discriminant analysis being linearly combined in order to obtain classification functions. Classification results were subsequently analyzed to establish whether the misclassified pears presented values clearly different from the rest of their corresponding group (sound or disordered) that may underlie the classification error.

\subsubsection{Metrology}

The repeatability error was computed for each feature as the average standard deviation (STD) over the values extracted from the three acquired repetitions, and the variation coefficient was then calculated as the average STD per fruit divided by the absolute value of the corresponding mean.

\subsubsection{Microscopic and macroscopic references}

Optical microscopy was undertaken on very thin slices for the studied tissues (Olympus BX60 with the $10 \times$ and $20 \times$ lens) in order to obtain a closer inspection of the tissues structure.

As for on-line experiments, pear firmness was assessed by means of a manual penetrometer (plunger diameter $8 \mathrm{~mm}$ ). Two measurements were performed per pear near the equatorial zone and the averaged value was obtained. Afterwards, the pears were cut in half in order to expose the same slice imaged by MRI to a RGB image capture with a digital camera. Segmentation through the red and blue components allowed the computation of the percentage of disordered-area (core cavities included) in relation to the whole captured tissue. Soluble solids were measured with a digital refractometer (PR-101 ATAGO) as a reference for sugar content. Two measurements were obtained per fruit by squeezing both halves and the resulting values were averaged.

\section{Results and discussion}

\subsection{Microscopic study}

\subsubsection{Analysis of the effect of variable $90-180^{\circ}$ pulse spacing on CPMG sequence.}

Variable $90-180^{\circ}$ pulse spacing $(\tau)$ on the transverse relaxation time $\left(T_{2}\right)$ showed the expected effect of decreasing the transverse relaxation rate $\left(1 / T_{2}\right)$ when pulsing frequency $(1 / \tau)$ is increased (results not shown). This effect is accounted for by the loss of phase coherence between processing protons caused by the proton switching between sites differing slightly in resonance frequency (Hills, 1998). Thus, when the pulsing frequency is much greater than the proton exchange rate, the dephasing is completely refocused by the train of $180^{\circ}$ pulses, so proton exchange has no additional dephasing effect on the $T_{2}$ computation achieving a minimum for $1 / T_{2}$ at very high pulsing frequency $(5000 \mathrm{~Hz}$ for our experimental design). When the pulsing rate is lower than the proton exchange rate, the frequency variation experienced by the proton causes a rapid dephasing of its transverse magnetization increasing the transverse relaxation rate $\left(1 / T_{2}\right)$ for pulsing rate lower than $1000 \mathrm{~s}^{-1}$ (results not shown).

The microstructure of the tissue samples also plays a role on the transverse relaxation rate $\left(1 / T_{2}\right)$ as suggested by the results (not shown), since the diffusive exchange rate of protons between compartments, which affects $T_{2}$, depends on cell morphology and membrane permeability (Hills, 1998). Such exchange is normally several orders slower than the proton exchange rate between chemical sites so that the ditfusive dispersion dominates at the shortest pulse spacing rates $(1 / \tau)$ increasing the transverse relaxation rate $\left(1 / T_{2}\right)$, as was seen for both sound and disordered pear tissues (results not shown). At long pulse spacing rates dispersions of the different tissues converge though the transverse relaxation times of the disordered tissues are higher than those of the corresponding sound ones at any pulse spacing (results not shown).

When tissue compartmentation is destroyed by means of juice extraction, the water diffusivity effect on $T_{2}$ disappears, and so a plateau was found for low ( $1 / \tau$ ) (results not shown). Comparison between pear tissues and juice reveals that transverse relaxation rate $\left(1 / T_{2}\right)$ is greatly affected by the pear tissue microstructure (since free juice has much lower ( $\left.1 / T_{2}\right)$ than tissue). Results also show that high magnetic field strength enhances relaxation rate $\left(1 / T_{2}\right)$ as higher values are obtained for $300.15 \mathrm{MHz}$ compared to $100.13 \mathrm{MHz}$ (results not show11).

Optical microscopy (Fig. 1) confirmed that for damaged tissues, the cells loose their natural angular morphology and even their integrity, together with the loss of compactness and coherence for the whole tissue. Accumulation of vesicles containing brown-coloured compounds is also revealed by the microscopic images in Fig. 1. Such vesicles were expected according to Kader (1989) on flesh browning in 'Bartlett' pears. Thus, the $T_{2}$ decrease associated with disordered tissues can be addressed to an effect of diffusion related to the loss of membrane integrity that causes simultaneous magnification of 

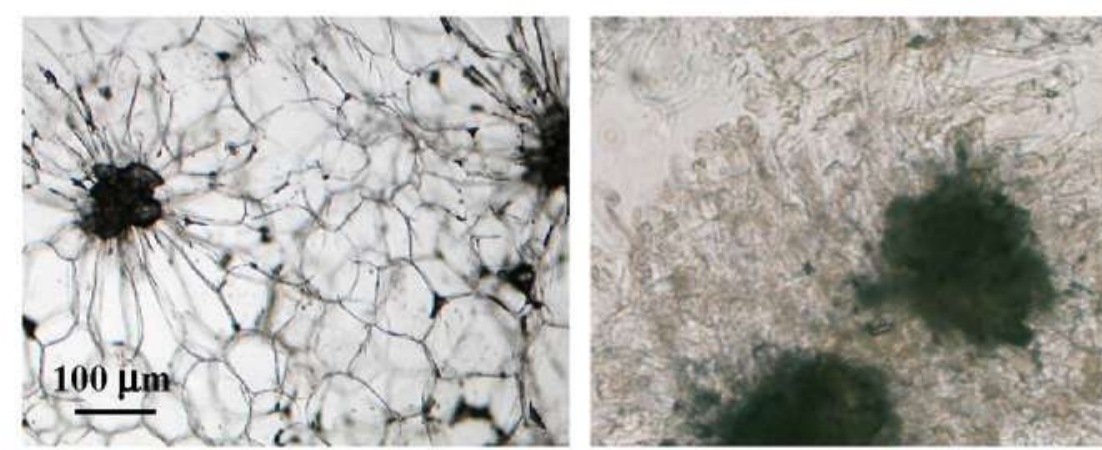

Fig. 1. Optical microscopy images of sound flesh (left) and disordered flesh (right). Tissue disintegration and brown vesicle accumulation is visible.

(a) 2D Laplace transform of FT-200-300MHZ-JUNE2 (Log intensity)

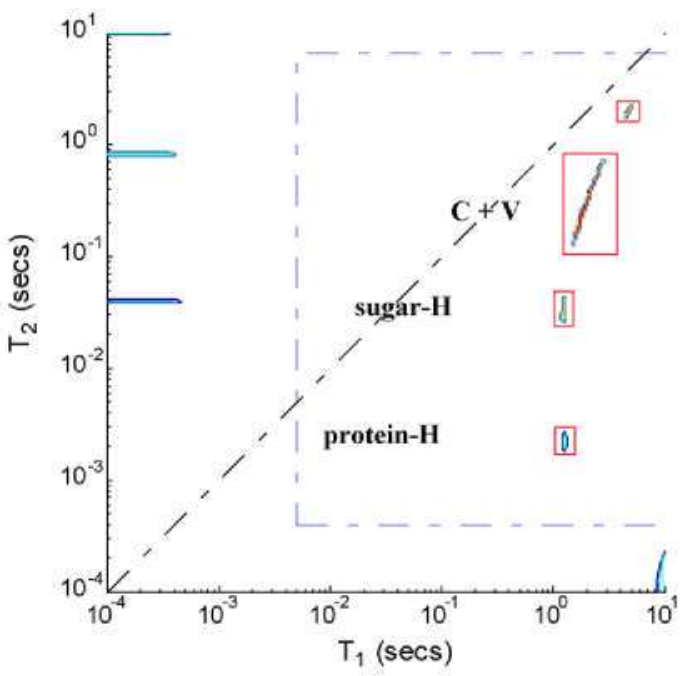

(c)

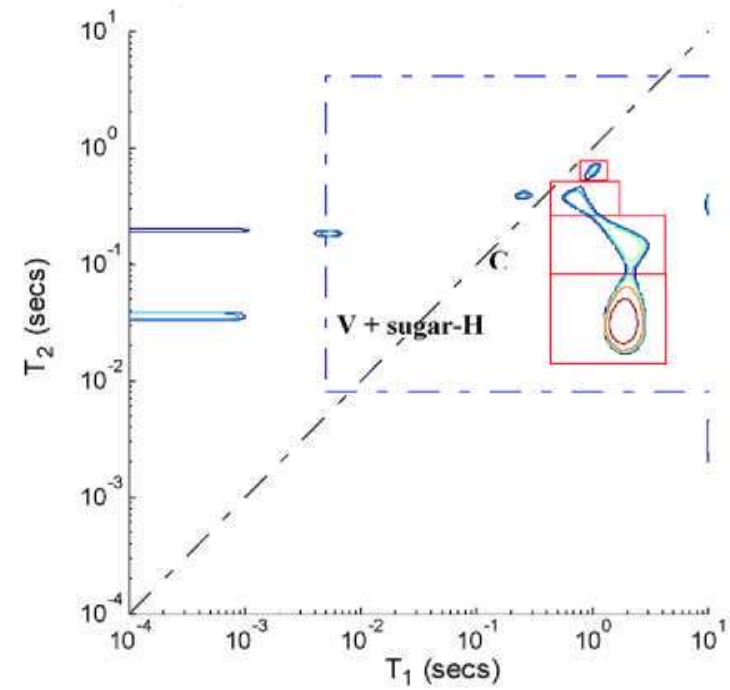

(b) $2 \mathrm{D}$ Laplace transform of DT-200-300MHZ-MAY29 (Log intensity)

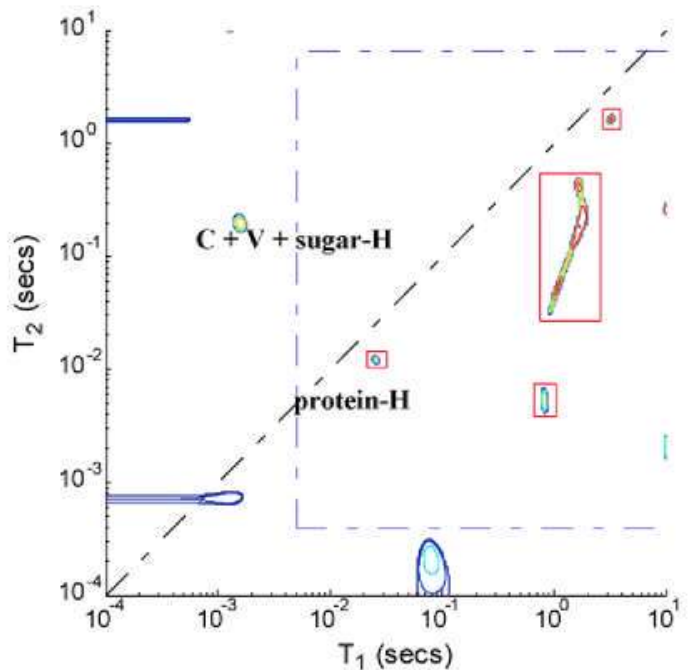

(d) $2 \mathrm{D}$ Laplace transform of DT-4000-300MHZ-MAY29 (Log intensity)

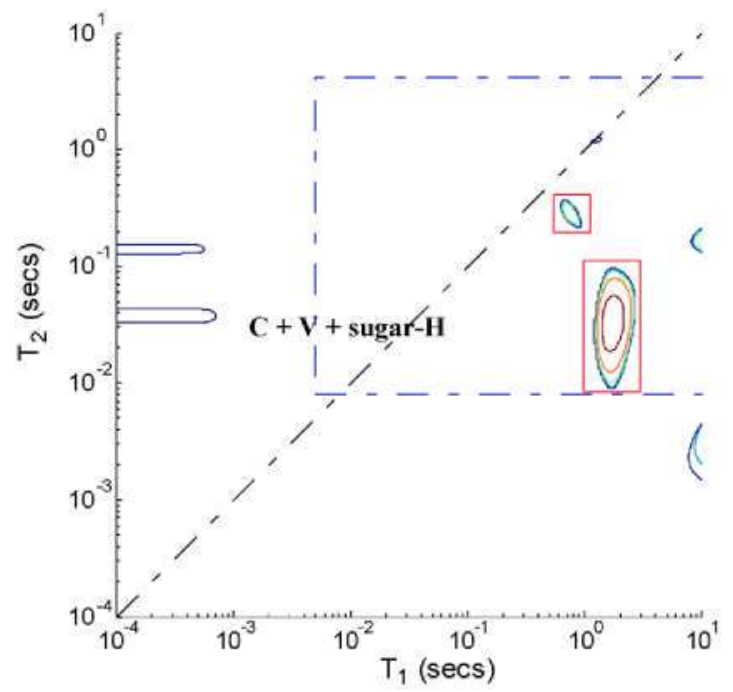

Fig. 2. $T_{1}-T_{2}$ correlation spectra obtained from both sound ( $\mathrm{a}$ and $\mathrm{c}$ ) and disordered (b and d) flesh at high magnetic field strength ( $300.15 \mathrm{MHz}$ resonance frequency) with a $90-180^{\circ}$ pulse spacing of $200 \mu \mathrm{s}$ ( $\mathrm{a}$ and b) and $4000 \mu \mathrm{s}$ ( $\mathrm{c}$ and d). The dashed rectangle shows the limits of reliable values set by the pulse sequence spacings. The dashed diagonal indicates the condition $T_{1}=T_{2} . \mathrm{C}$ stands for water protons in cytoplasm, $\mathrm{V}$ stands for water protons vacuole, sugar- $\mathrm{H}$ and protein- $\mathrm{H}$ stand for protons in sugar and protein molecules, respectively. 
the local gradients due to the magnetic susceptibility discontinuity across the phenolic vesicles-water interface. This effect is enhanced at higher magnetic field strength as local gradients are proportional to the applied magnetic field. These results were the basis for following experiments on high resolution NMR.

\subsubsection{Analysis of $\left(T_{1}-T_{2}\right)$ correlation spectra}

The $\left(T_{1}-T_{2}\right)$ cross-correlation spectroscopy shown in Fig. 2 provides quantitative information about the water compartmentation, as water in different subcellular organelles is often characterized by different proton relaxation times $T_{1}$ and $T_{2}$ (Hills and Remigereau, 1997; Hills and Nott, 1999). The anal$y$ sis of the spectra also provides qualitative information on the cell structure integrity.

Fig. 2 also shows that at any pulse spacing used in this study (200 and $4000 \mu \mathrm{s}$ ), the patterns of the sound and the disordered tissues are comparable. For sound tissue (Fig. 2a) different pro-
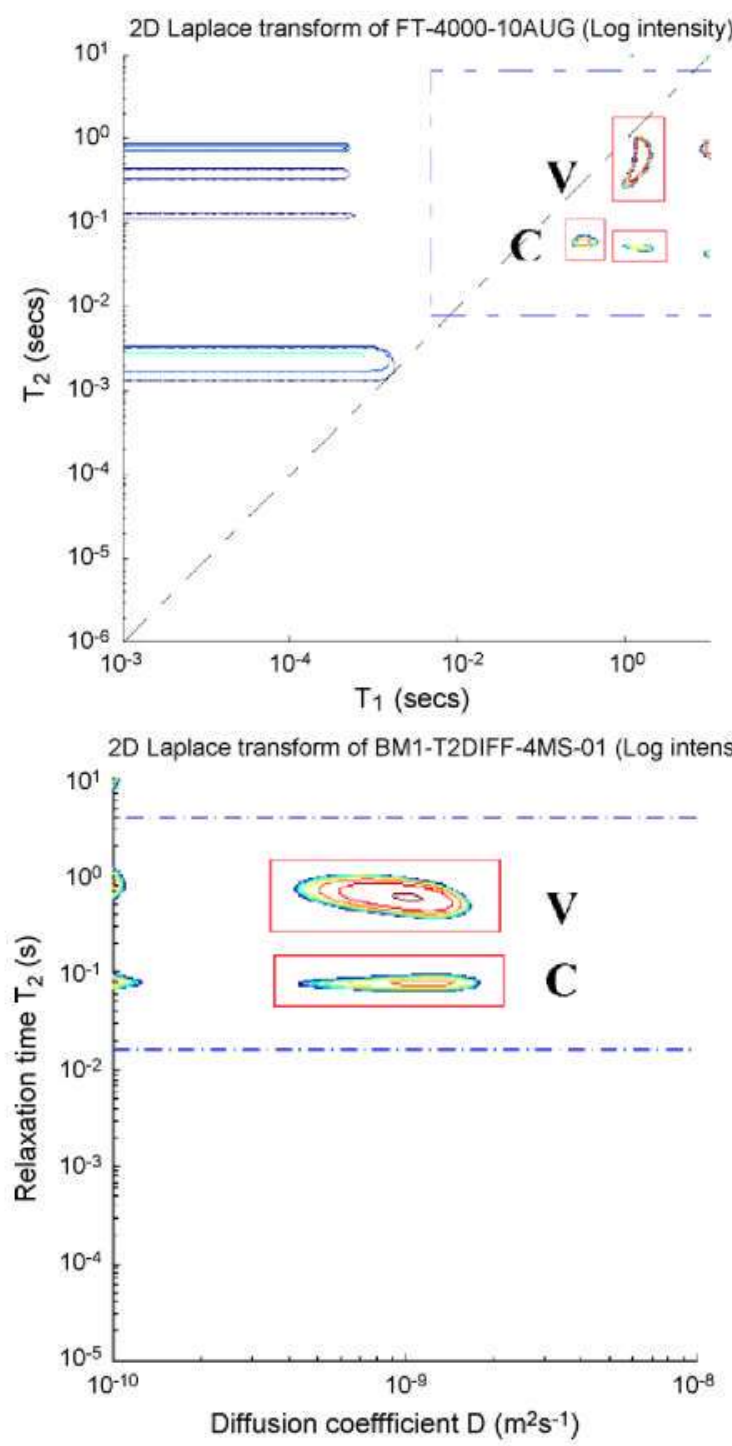

ton pools can be distinguished. A preliminary assignment can be done, according to previous studies on parenchyma tissue of apple (Hills and Remigereau, 1997), which concludes that water is normally compartmentalised into vacuole, cytoplasm and extracellular space with increasing mobility restriction, respectively, that is, decreasing $T_{2}$ values. The dominant peak (around $1768 \mathrm{~ms} T_{1}, 221 \mathrm{~ms} T_{2}$ ) is tentatively associated with water in vacuole and water in cytoplasm (see labelled areas in Fig. 2). The dispersion in the $T_{2}$ values makes it difficult to resolve clearly both compartments. Such dispersion is accounted for by the water diffusive exchange and the distribution in cell and compartment size. Smaller peaks in Fig. 2a may correspond to protons in sugar ( $\left.1250 \mathrm{~ms} T_{1}, 35 \mathrm{~ms} T_{2}\right)$ and protein ( $1250 \mathrm{~ms} T_{1}$, $2 \mathrm{~ms} T_{2}$ ) molecules, although this labelling is made at a higher level of uncertainty.

As for disordered flesh (Fig. 2b), the proton pools are grouped into a lower number of populations, since cytoplasm and vacuole decrease in average $T_{2}$ and apparently merge with the sugar peak

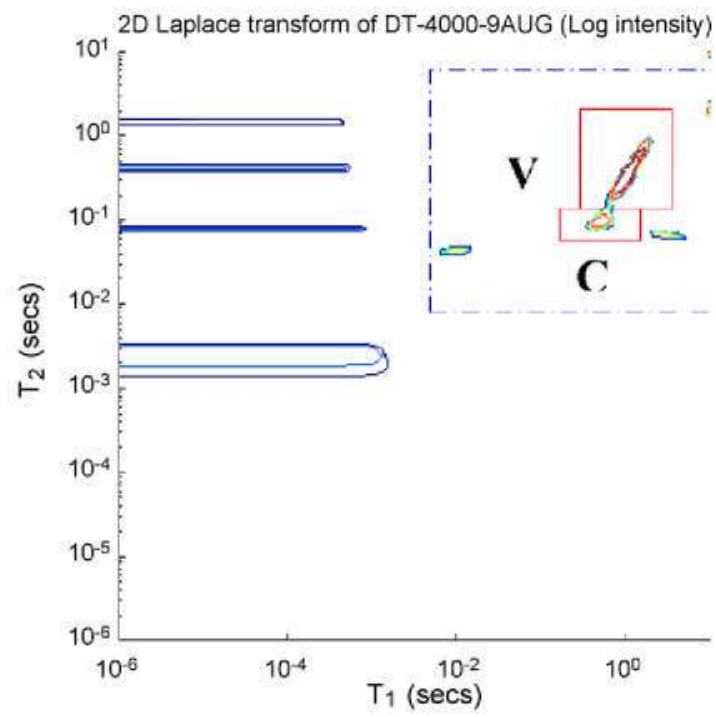

2D Laplace transform of BM1-T2DIFF-4MS-01 (Log intensity)

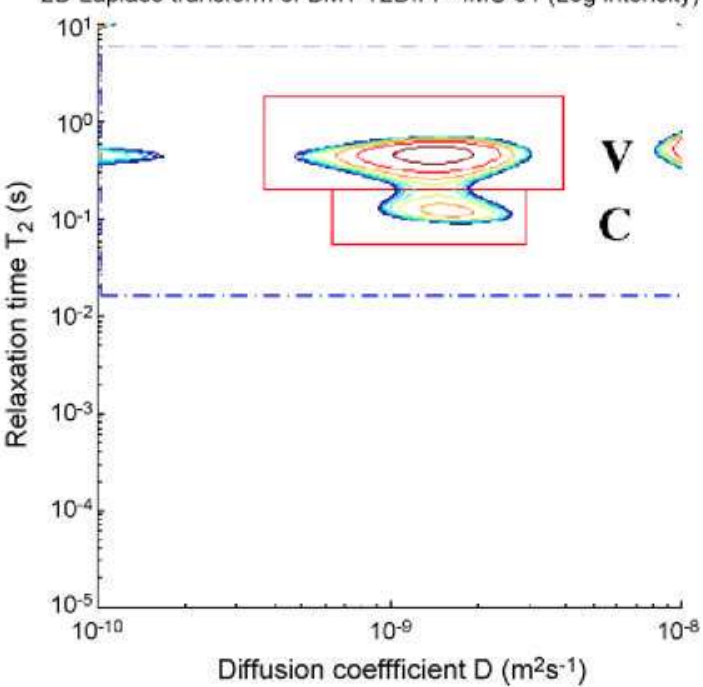

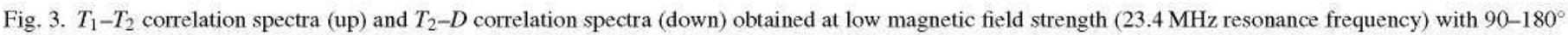

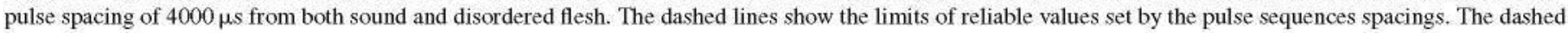
diagonal indicates the condition $T_{1}=T_{2}$. C stands for water protons in cytoplasm, $\mathrm{V}$ stands for water protons in the vacuole. 
(see labelled areas), highlighting the enhancement of the diffision exchange and hence, the loss of membrane integrity. The water diftusion through strong internal field gradients arising from susceptibility discontinuities may also drive the observed $T_{2}$ shortening.

Also in Fig. 2, a study of the effect of two CPMG pulse spacing $(\tau), 200$ and $4000 \mu$ s (upper and lower row, respectively) on $T_{2}$ shows that at high magnetic field strength $(300.15 \mathrm{MHz})$, major differences between sound and disordered tissue occur for long $\tau$ values $(4000 \mu \mathrm{s})$. Fig. $2 \mathrm{c}$ and $\mathrm{d}$ corresponds to $\left(T_{1}-T_{2}\right)$ cross-correlation spectra for sound and disordered tissues, it being noticeable that for disordered tissues, the peaks for different compartments completely merge.

Fig. 3 shows $\left(T_{1}-T_{2}\right)$ cross-correlation spectra at low magnetic field strength (upper row) together with $\left(T_{2}-D\right)$ correlation spectroscopy (lower row). Measurements shown in the upper row of Fig. 3 were performed at low magnetic field strength (23.4 MHz resonance frequency) and with long pulse spacing $(4000 \mu s)$ in order to achieve a better characterization of the tissues. ( $T_{2}-D$ ) correlation spectroscopy is used for associating the $T_{2}$ components with their corresponding water diffusion coefficients, $D\left(\mathrm{~m}^{2} / \mathrm{s}\right)$. In this study, correlation between $T_{2}$ and D was performed to assess the level of membrane integrity loss of the main cell compartments for disordered pears. According to some references, $\left(T_{2}-D\right)$ correlation spectroscopy can be used to monitor microscopic sub-cellular changes as shown during ripening of banana (Ratio et al., 2005) or for avocado (Marigheto et al., 2005). In our study, the diffusion effect on $T_{2}$ dispersion is showed at long pulse spacing $(4000 \mu s)$, which agrees with results obtained for pulse spacing effect on tissues. Besides, at low magnetic field strength $(23.4 \mathrm{MHz}$ ), the local magnetic field gradients are significantly reduced, limiting the enhancement of the dephasing in the transverse magnetization.

The lower row in Fig. 3 shows the graphs corresponding to the $\left(T_{2}-D\right)$ correlation of sound and disordered tissue, respectively. In these graphs two dominant peaks are found which have been associated with the water in the vacuole (long $T_{2}$ ) and in the cytoplasm (shorter $T_{2}$ ). For the former peak, $T_{2}$ decreases to values of about $600 \mathrm{~ms}$ for sound tissue to values of about $450 \mathrm{~ms}$ in damaged tissue (Table 2), in good agreement with that observed in the $\left(T_{1}-T_{2}\right)$ correlation spectra (Fig. 2 ). For the water diffusion coefficient $(D)$, it is observed that the value increases for both compartments in damaged tissue. Table 2 shows that the averaged $D$ value for the vacuole is $1.02 \times 10^{-9} \mathrm{~m}^{2} / \mathrm{s}$ for sound tissue and $1.39 \times 10^{-9} \mathrm{~m}^{2} / \mathrm{s}$ for disordered tissue. As for the cytoplasm, average $D$ increases from $9.68 \times 10^{-10}$ to $1.48 \times 10^{-9} \mathrm{~m}^{2} / \mathrm{s}$. Such results would explain both the shortened transverse relaxation times $\left(T_{2}\right)$ and the peaks merging due to the enhancement of the water diffusion exchange between compartments. The microscopy results have been used to contrast the macroscopic analysis of internal browning in pears.

\subsection{Macroscopic study}

In order to characterize the macroscopic relaxation properties of pear tissues, a preliminary analysis of $T_{2}$ maps of whole pears corresponding to $0.47 \mathrm{~mm}$ /pixel spatial resolution were performed at LPF-UCM (Fig. 4). The average $T_{2}$ value for unaffected voxels $(0.47 \mathrm{~mm} \times 0.47 \mathrm{~mm} \times 5 \mathrm{~mm})$ was $56 \pm 5 \mathrm{~ms}$. Note that this value is lower than that obtained at the microscopic level due to overall tissue averaging ('partial volume effect'). Over the time course of the disorder development, damaged tissue ( $36 \pm 5 \mathrm{~ms}$ average $T_{2}$ ) reached $T_{2}$ values below those of sound tissue, with a decrease up to $50 \%$. However, it is worth noting that within the disordered regions, voxels of tissue were found with average macroscopic $T_{2}$ values higher than those of sound ones, $67 \pm 4 \mathrm{~ms}$. Although these previous data were extracted from a very low number of samples (three fruit), these results support the hypothesis proposed by Lammertyn et al. (2003a) on the loss of cell membrane integrity that leads to an increase in the free water level at the first stage, and subsequent evaporation. This hypothesis was considered to withstand the opposite results obtained for 'Bartlett' pears (Wang and Wang, 1989) and 'Conference' pears (Lammertyn et al., 2003a), regarding the higher free water content of the disordered tissue in such varieties when compared to sound tissues.

'Blanquilla' pear data, shown in Fig. 4, indicate that water protons would undergo at a first stage an increase in mobility revealed at the macroscopic level as a higher average transverse relaxation time $\left(T_{2}\right)$ within the voxel. This proton pool would be more susceptible to evaporation when a relative humidity imbalance of the storage atmosphere led to moisture diffusion. Moreover, water transport would be enhanced by the higher water diffusion coefficients observed at the microscopic level for damaged tissues. In agreement with Veltman et al. (2003), the cell decompartmentation showed by the optical microscopy images, and highlighted with high spatial resolution NMR, facilitates the accessibility of polyphenoloxidase to its substrate with

Table 2

Results of the $T_{2}-D$ correlation spectra for the integrated peaks of three pears

\begin{tabular}{|c|c|c|c|c|c|c|c|}
\hline \multirow[t]{2}{*}{ Fruit sample } & \multirow[t]{2}{*}{ Compartment } & \multicolumn{3}{|l|}{ Sound tissue } & \multicolumn{3}{|l|}{ Disotdered tissue } \\
\hline & & $D\left(\mathrm{~m}^{2} / \mathrm{s}\right) \times 10^{-9}$ & $T_{2}$ (ns) & Area $(\%)$ & $D\left(\mathrm{~m}^{2} / \mathrm{s}\right) \times 10^{-9}$ & $T_{2}$ (ms) & Area (\%) \\
\hline 1 & $\mathrm{~V}$ & 1.09 & 597 & 86.2 & 1.35 & 452 & 93.7 \\
\hline 1 & $C$ & 1.19 & 78 & 13.8 & 1.39 & 71 & 6.3 \\
\hline 2 & $\mathrm{~V}$ & 0.99 & 625 & 86.5 & 1.44 & 452 & 85.8 \\
\hline 2 & C. & 0.86 & 75 & 13.4 & 1.57 & 118 & 14.2 \\
\hline 3 & $\mathrm{~V}$ & 1.04 & 570 & 96.3 & 1.39 & 474 & 100 \\
\hline 3 & C. & 0.85 & 90 & 3.7 & Merged & Merged & Merged \\
\hline
\end{tabular}

The compartments identified in Fig. 5 correspond to fruit sample 1. V stands for vacuole and C. for cytoplasm. 

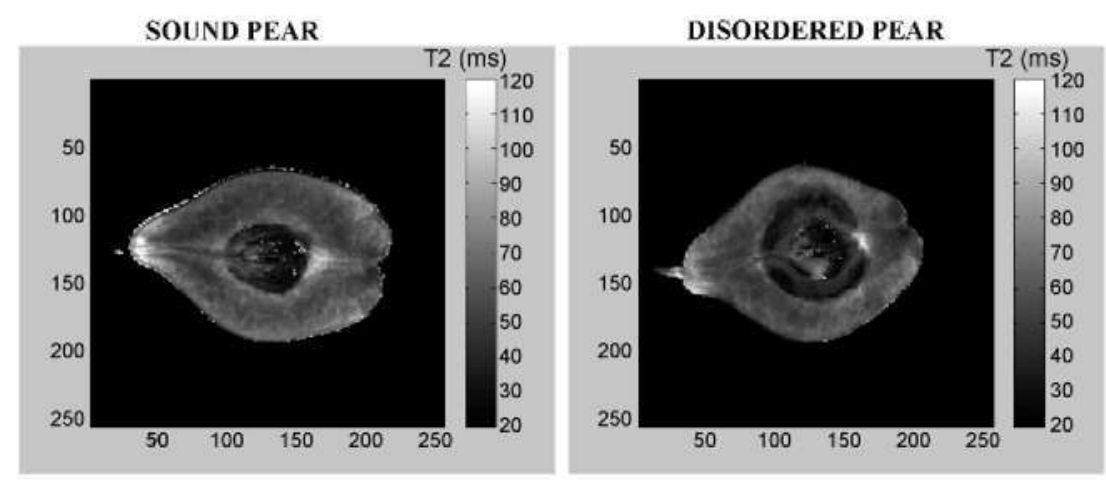

Fig. 4. $T_{2}$ maps of the longitudinal section of a sound pear (left) and pear with developed disorder (right).

the consequent accumulation of the brown phenolic vesicles, as shown in the microscopic images in Fig. 1.

In view of the above mentioned results on macroscopic $T_{2}$ maps, it was decided $T_{2}^{*}$-weighted as well as PD-weighted MR images (related to water content and hence to evaporation) would provide useful information on internal breakdown development since the signal intensity would be affected by the effects of the disorder on the tissues. Such an expectation was confirmed by fast stationary imaging, which showed a noticeable contrast between tissues (results not shown).

For $T_{2}^{*}$-weighted images obtained from longitudinal tomography of stationary fruit and RGB images of cut samples, high correspondence was found between the hypo-intense signal regions (short $T_{2}$ ) and the brown coloured tissue in the RGB pictures (results not shown). Within disordered regions, high intensity signal pixels were also displayed in good accordance with previous $T_{2}$ maps shown in Fig. 4 .
For dynamic inspection ( 32 pears), the field of view was shifted so as to obtain transverse instead of longitudinal tomography. Such a decision was supported by the results shown by Lammertyn et al. (2003b) in 'Conference' pears. For transverse pear sections, the disordered tissue appeared concentrically distributed around the core as well as gathered at specific locations (results not shown), a fact that had already been highlighted by the previous authors.

The reference values of the set of conveyed pears are shown in Table 3 . Five pears out of the 16 (previously sorted as potentially disordered) showed no disorder development. For disordered pears, the range of brown tissue percentages varied from 12 to $83 \%$. These values include the core cavities housing the seeds (when they appeared), whose extent varies from less than $1-4 \%$. Fruit firmness (ranging from 9.4 to $66.4 \mathrm{~N}$ ) was not correlated with the percentage of brown area. Soluble solids contents also were not correlated with the extent of the brown

Table 3

Reference measurements of fruit used in the on-line experiments

\begin{tabular}{|c|c|c|c|c|c|c|c|}
\hline \multirow[t]{2}{*}{ Sample } & \multicolumn{3}{|l|}{ Sound pears } & \multirow[t]{2}{*}{ Sample } & \multicolumn{3}{|l|}{ Disordered pears } \\
\hline & Hypo-intense area $(\%)$ & Firmness $(\mathrm{N})$ & $\begin{array}{l}\text { Soluble solids } \\
\text { content }(\%)\end{array}$ & & Hypo-intense area $(\%)$ & Firmness $(\mathrm{N})$ & $\begin{array}{l}\text { Soluble solids } \\
\text { content (\%) }\end{array}$ \\
\hline $\mathrm{H} 1$ & 2.4 & 9.8 & 14.0 & Al & 12.0 & 19.0 & 14.4 \\
\hline $\mathrm{H} 2$ & 3.1 & 11.4 & 14.4 & $\mathrm{~A} 2$ & 20.6 & 18.9 & 12.9 \\
\hline H3 & 3.6 & 12.7 & 13.8 & $\mathrm{~A} 3$ & 30.6 & 17.4 & 12.7 \\
\hline $\mathrm{H} 4$ & 4.1 & 12.7 & 13.2 & A4 & 31 & 17.1 & 14.2 \\
\hline H5 & 2.9 & 14.1 & 14.8 & A5 & 37.5 & 16.3 & 10.8 \\
\hline H6 & $<1$ & 16.2 & 14.1 & A6 & 39.2 & 16.8 & 12.9 \\
\hline $\mathrm{H} 7$ & 2.3 & 16.3 & 11.5 & A7 & 44.6 & 17.6 & 13.4 \\
\hline $\mathrm{H} 8$ & 3.1 & 16.6 & 14.4 & A 8 & 45.2 & 17.9 & 14.3 \\
\hline H9 & $<1$ & 17.1 & 14.8 & A9 & 49.0 & 17.3 & 13.0 \\
\hline H10 & 3.5 & 17.4 & 12.2 & A 10 & 64.0 & 13.9 & 13.2 \\
\hline H11 & 3.4 & 17.9 & 14.2 & A11 & 83.0 & 9.4 & 13.5 \\
\hline $\mathrm{H} 12$ & $<1$ & 20.3 & 15.6 & & & & \\
\hline $\mathrm{H} 13$ & $<1$ & 21.7 & 12.0 & & & & \\
\hline $\mathrm{H} 14$ & 3.9 & 28.5 & 13.7 & & & & \\
\hline H15 & 3.8 & 46.3 & 12.4 & & & & \\
\hline $\mathrm{H} 16$ & 3.6 & 48.2 & 13.2 & & & & \\
\hline H17 & 3.5 & 48.4 & 13.7 & & & & \\
\hline H18 & 2.1 & 49.6 & 13.1 & & & & \\
\hline H19 & 4.2 & 50.8 & 12.7 & & & & \\
\hline $\mathrm{H} 20$ & 2.6 & 62.1 & 12.7 & & & & \\
\hline $\mathrm{H} 21$ & 3.8 & 66.4 & - & & & & \\
\hline
\end{tabular}

Hypo-intense area stands for disordered tissue areas and core cavities segmented in the RGB images and related to the whole section. 

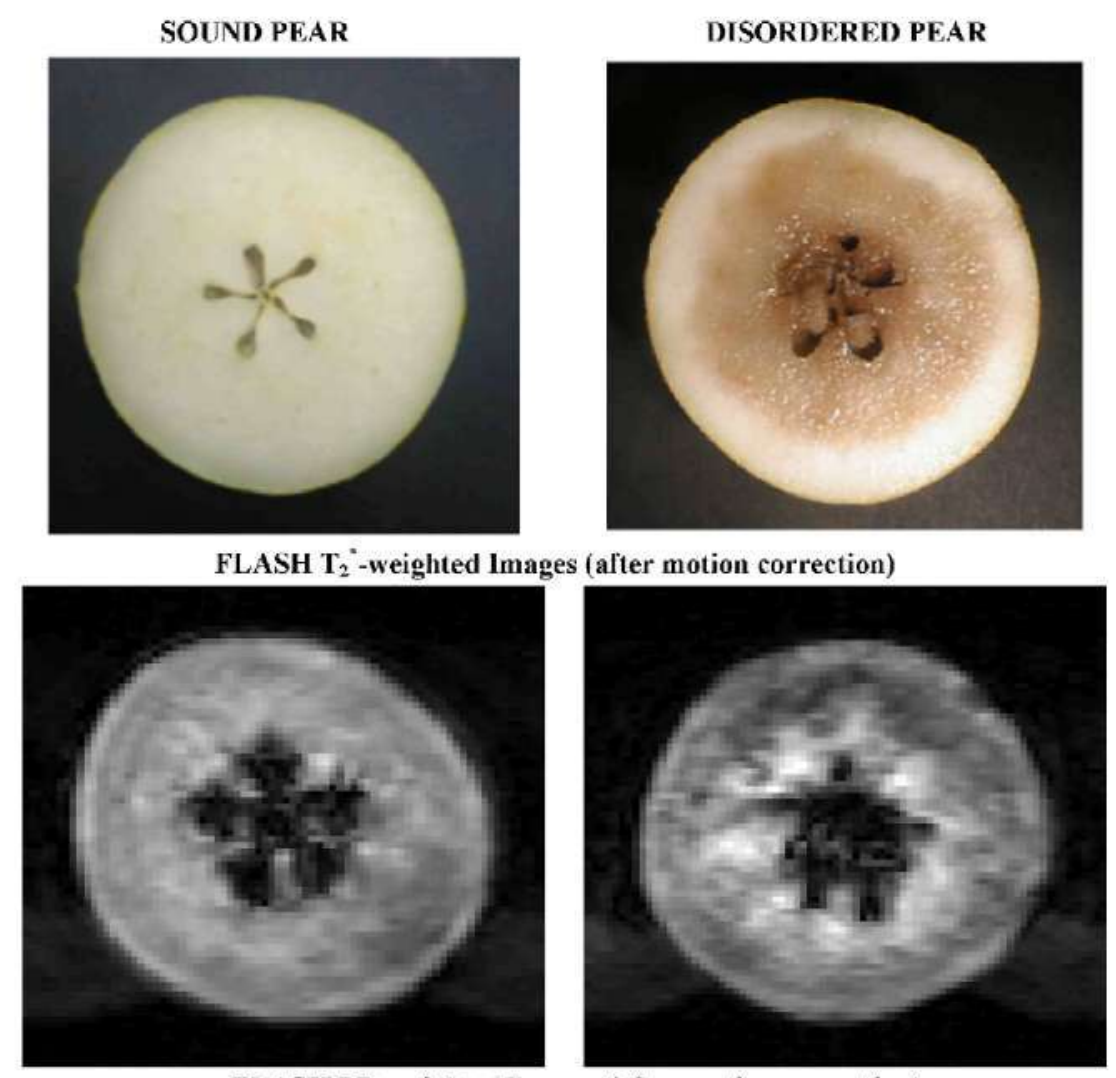

FLASH PD-weighted Images (after motion correction)

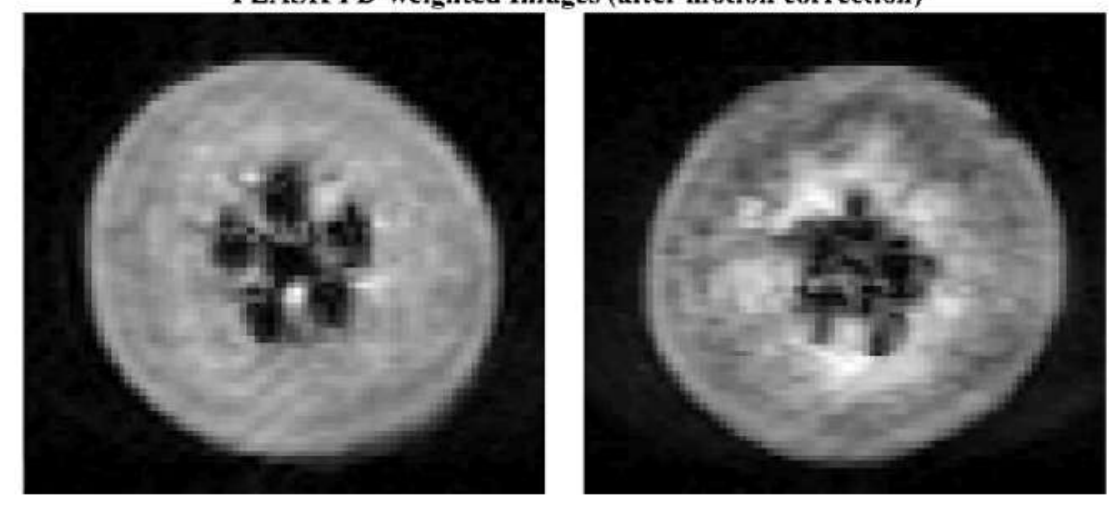

Fig. 5. MRI for macroscopic on-line identification. FLASH $T_{2}^{*}$-weighted image (middle) and FLASH PD-weighted image (bottom) after motion correction acquired at $54 \mathrm{~mm} / \mathrm{s}$ belt speed.

area in any case. Therefore, these results along with the lack of external symptoms reinforce the need for internal inspection of fruit.

Pears were imaged while conveying at $54 \mathrm{~mm} / \mathrm{s}$. Images were reconstructed after correcting the motion effect (Fig. 5). The quality of the image reconstruction was satisfactory since high correspondence with the RGB pictures was observed (see upper row in Fig. 5).

The first step in the automated image analysis was to extract the signal intensity histogram from the region of interest that is the fruit section (ROI). For sound pears, the tissue homogeneity is displayed as narrow histograms of the MR images. Pixels with low signal intensity are distributed nearly homogenously among the lowest histogram categories (Fig. 6). These categories include those pixels corresponding to the core cavities. As for the disordered pears, the histograms of both types of images, $T_{2}^{*}$ and PD-weighted, show a broadening of the main population of pixels. An increase is detected in the number of pixels within extreme categories (highest and the lowest signal intensity) (Fig. 6). Core cavities were included in the analysis without labelling owing to the difficulty of automatically differentiating them from the disordered tissue, since both regions show hypo-intense signal. For the PD-weighted images the shift of the histogram towards classes of pixels with lower intensity signal values highlights the loss of proton density due to water evaporation underlying the disorder development (Fig. 6) outlined in the microscopic study.

The differences between histograms of sound and disordered pears were exploited by extracting several descriptive features. 


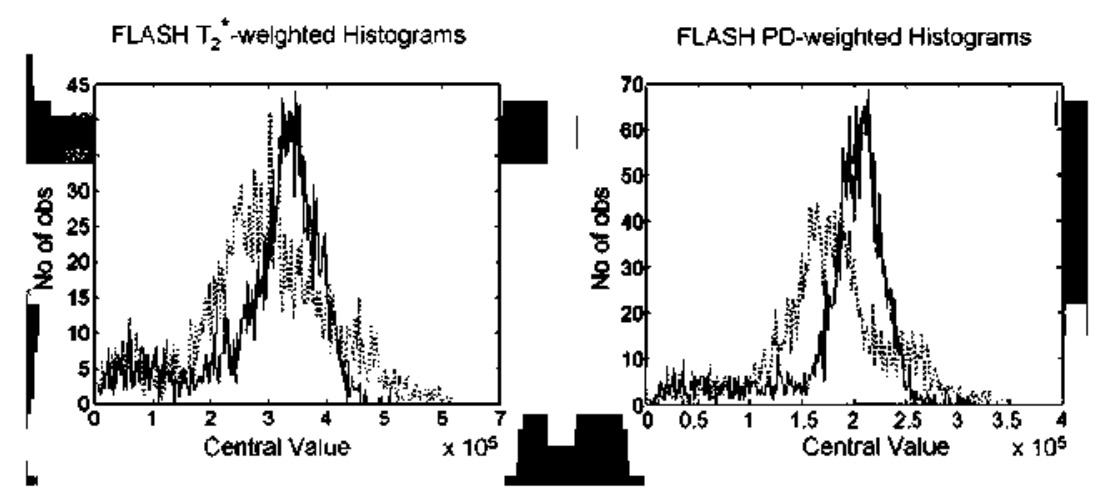

Fig. 6. Examples of signal intensity histograms of region of interest segmented from FLASH $T_{2}^{*}$-weighted image (left) and from FLASH PD-weighted image (tight) of sound (solid line) and disordeted pears (dotted line).

Table 4

Descriptive statistics and ANOVA results for segmented FLASH images of sound and disordered pears

\begin{tabular}{|c|c|c|c|c|c|c|c|c|c|c|}
\hline & \multicolumn{5}{|c|}{ FLASH $T_{2}^{*}$-weighted } & \multicolumn{5}{|c|}{ FLASH PD-wejghted } \\
\hline & \multicolumn{2}{|l|}{ Sound } & \multicolumn{2}{|c|}{ Disordered } & \multirow{2}{*}{ Significance } & \multicolumn{2}{|l|}{ Sound } & \multicolumn{2}{|c|}{ Disordered } & \multirow[t]{2}{*}{ Significance } \\
\hline & Mean & STD & Mean & STD & & Mean & STD & Mean & STD & \\
\hline SCV & 27.55 & 5.34 & 34.65 & 5.29 & $* *$ & 22.71 & 4.28 & 28.58 & 4.05 & ** \\
\hline Skewness & -0.92 & 0.61 & 0.28 & 0.56 & * & -1.25 & 0.52 & -0.02 & 0.52 & N: \\
\hline Kurtosis & 1.26 & 0.82 & 0.81 & 0.87 & n.s. & 2.70 & 1.14 & 0.92 & 0.58 & $* *$ \\
\hline C3 & 0.1 & 0.1 & 0.2 & 0.1 & n.s. & 0.1 & 0.1 & 0.1 & 0.1 & n.s. \\
\hline C26 & 2.4 & 1.4 & 3.1 & 2.1 & n.s. & 1.4 & 0.9 & 1.7 & 1.1 & n.s. \\
\hline $\mathrm{C} 64$ & 7.5 & 2.9 & 12.6 & 5.2 & ** & 4.7 & 2.3 & 7.0 & 3.0 & $+*$ \\
\hline $\mathrm{Cl} 28$ & 33.5 & 16.8 & 72.0 & 15.5 & $* *$ & 23.3 & 16.4 & 61.1 & 19.1 & $* *$ \\
\hline $\mathrm{Cl} 92$ & 9.4 & 5.5 & 6.5 & 4.8 & $*$ & 9.3 & 9.7 & 5.7 & 4.5 & n.s. \\
\hline $\mathrm{C} 230$ & 5.9 & 6.4 & 6.2 & 6.8 & n.s. & 5.8 & 6.3 & 5.5 & 5.9 & n.s. \\
\hline $\mathrm{C} 253$ & 5.9 & 6.7 & 6.3 & 7.0 & n.s. & 6.3 & 6.7 & 5.7 & 6.2 & n.s. \\
\hline$N$ & \multicolumn{2}{|c|}{52} & \multicolumn{2}{|c|}{28} & & \multicolumn{2}{|c|}{63} & \multicolumn{2}{|c|}{33} & \\
\hline
\end{tabular}

All parameters are dimensionless ( ${ }^{*}$ stands for significance at $5 \%$, ${ }^{* *}$ stands for significance at $1 \%$, n.s. stands for no signiticance).

Table 4 shows the most relevant statistics for the features defined: $\mathrm{SCV}$, skewness, kurtosis, C3, C26, C64, C128, C192, C230 and C253. The number of samples for FLASH $T_{2}^{*}$-weighted images is lower than for PD-weighted since (16 out of 96) images had to be excluded from the analysis due to signal acquisition errors.

For $T_{2}^{*}$-weighted images $(N=80)$ significant difterences between sound and disordered pears were found for $\mathrm{SCV}$, skewness, $\mathrm{C} 64, \mathrm{C} 128$ and $\mathrm{Cl} 92$ (Table 4), yet the features did not allow the extraction of individual thresholds for each parameter in order to identify non-disordered fruit. A forward stepwise discriminant analysis was then performed, and SCV, skewness, $\mathrm{C} 64$ and $\mathrm{C} 128$ were selected to generate the classification functions. Correct classifications of pears into sound and disordered categories (98 and $86 \%$, respectively) were achieved (Table 5).

As for $\mathrm{PD}$-weighted images, significant differences were found for SCV, skewness, kurtosis, C64 and $\mathrm{C} 128$ (Table 5). The set of individual thresholds addressed for sound pears (SCV $\leq 21$; skewness $\leq-1.1$; kurtosis $\geq 2.5$; C64 $\leq 1.5$; and Cl28 $\leq 24)$ resulted in a percentage of correct classification of $97 \%$ for sound and $91 \%$ for disordered pears. In comparison, the forward stepwise discriminant analysis used SCV, skewness, kurtosis and C64 leading to a 98.4 and a $91 \%$ of correctly classi- fied fruit for sound and disordered fruit, respectively (Table 5). The fruit showing a minimum extent of damage (12\%) were always correctly classified with the discriminant functions.

The study of the repeatability showed that the features extracted from PD images are more consistent than those from the $T_{2}^{*}$-weighted as stated by their lowest coefficient of variation (results not shown). Features SCV, C64 and C128 present the highest repeatability, conferring robustness to the classification functions since such features were selected in the

Table 5

Classification results of the discriminant analysis

\begin{tabular}{lcccc}
\hline & Percent correct & Sound & Disordered & Total \\
\hline \multicolumn{2}{l}{ FLASH $T_{2}^{*}$-weighted } & & & \\
Sonnd & 98.1 & 51 & 1 & 52 \\
Disordered & 85.7 & 4 & 24 & 28 \\
Total & 93.8 & 55 & 25 & 80 \\
FLASH DP-weighted & 98.4 & 62 & 1 & 63 \\
Sonnd & 90.9 & 3 & 30 & 33 \\
Disordered & 95.8 & 65 & 31 & 96 \\
Total & & & & \\
\hline
\end{tabular}

Rows stand for observed classifications and columns stand for predicted classifications. 
discriminant analysis for both types of images. The rest of the features involved in the classification were not so repeatable.

\section{Conclusion}

In conclusion, internal browning in 'Blanquilla' pears is a postharvest disorder that may be identified with high resolution NMR and with MRI. The microscopic structure changes associated with disorder development are reflected in the NMR responses as differences in relaxometry $\left(T_{1}, T_{2}\right)$, diffusivity $(D)$ and proton density. Such changes can be regarded as responsible for the MR image contrast of whole fruit. The analysis of signal ( $T_{2}^{*}$ and proton density histograms) from whole fruit provides useful features for the identification of disordered pears.

The use of fast MRI sequences reduced the acquisition time to values lower than $1 \mathrm{~s}$ (703 and $484 \mathrm{~ms}$ for $T_{2}^{*}$ and PD-weighted images, respectively). Within this study it was possible to obtain dynamic images $(54 \mathrm{~mm} / \mathrm{s})$ and to correct motion artefacts. Reliable image reconstruction has been obtained for the fruit conveyed at $54 \mathrm{~mm} / \mathrm{s}$. Thus, an on-line procedure could achieve assessment of up to 30 pears $/ \mathrm{min}$ with $96 \%$ classification success for internal browning when using PD images.

In this study a minimum value of $12 \%$ of tissue disorder was always well identified. External validation will be required in the near future for assessing lower levels of damage. The potential for exploiting this result in a low-cost MRI imager is being explored as the high cost of commercial NMR spectrometers is one of the major factors hindering their development as on-line sensors (Hills, 2004, 2005).

\section{Acknowledgements}

We thank the Spanish Science and Education Ministry for the economical support, Jesús Ruiz-Cabello Osuna for valuable advices in this research, $\mathrm{M}^{\mathrm{a}}$ Encarnación Fernández for the technical support; and Dr. Mary Parker and Dr. Juan Bautista Martínez Laborde for assistance with optical microscopy. N. Marigheto gratefully acknowledges the support of a BBSRC CASE studentship.

\section{References}

Bracewell, R.N., 2000. The Fourier transform and its applications, third ed. McGraw-Hill Higher Education. Casson T.

Chen, P.. McCarthy. M.J., Kin. S.M.. Zion, B., 1996. Development of a highspeed NMR technique for sensing maturity of avocados. Trans. ASAE 39, 2205-2209.

Hernández-Sánchez, N., Barreiro, P., Ruiz-Altisent, M., Ruiz-Cabello, J., Fernández-Valle. M.E., 2004. Detection of freeze injury in oranges by magnetic resonance imaging of moving samples. Appl. Magn. Reson. 26, $431-445$.
Hernández, N., Barreiro, P., Ruiz-Altisent, M., Ruiz-Cabello, J., FetnándezValle, M.E. 2005. Detection of seeds in citrus using MRI undet motion conditions and inprovement with motion correction. Concept. Magn. Reson. B 26B, 81-92.

Hills, B.P., Remigereau, B., 1997. NMR studies of changes in subcellulat water compartmentation in parenchyma apple tissue during drying and freezing. Int. J. Food Sci. Tech. 32. 51-61.

Hills. B.P., Nott, K.P., 1999. NMR studies of water compartmentation in carrot parenchyma tissue during drying and freezing. Appl. Masn. Reson. 17, 521-535.

Hills. B.P. 1998. Magnetic Resonance Imaging in Fond Science. John Wiley \& Sons. New York.

Hills, B.P. 2004. Nuclear Magnetic Resonance Imaging. Chapter 9. In: Edwards, M. (Ed.), Detecting Foreign Bodies in Food, vol. 9. Woodhead Publishing Ltd., Cambridge, pp. 154-192.

Hills. B.P., 2005. Towards on-line MRI sensors. In: Engelsen, S.B., Belton. P.S., Jakobsen, H.J. (Eds.). Seventh Conference on Applications of Magnetic Resonance in Food Science. 13-15 September 2004, Copenhagen, Denmark, Pp. 175-185.

Hills. B.P., Benamira, S., Marigheto. N.. Wright, K.. 2004. $T_{1}-T_{2}$ correlation analysis of conplex foods. Appl. Magn. Reson. 26, 543-560.

Kadet, A.A., 1989. Mode of action of oxygen and carbon dioxide on postharvest physiology of "Bartlett" pears. Acta Hortic. 258, 161-167.

Lammertyn, J.. Aerts, M., Verlinden, B.E.. Schotsmans, W., Nicolai, B.M., 2000. Logistic regression analysis of factors influencing core breakdown in 'Conference' pears. Postharvest Biol. Technol. 20, 25-37.

Lammertyn, J., Dresselaers, T., Van Hecke, P., Jacsók. P., Wevers, M., Nicolai, B.M. 2003a. MRI and X-ray CT study of spatial distribution of core breakdown in 'Conference' pears. Magn. Res. Imag. 21. 805-815.

Lammertyn. J., Dresselaers, T., Van Hecke, P., Jacsók. P., Wevers, M., Nicolai, B.M., 2003b. Analysis of the time course of core breaktown in Conference" pears by means of MRI and X-ray CT. Postharvest Biol. Technol. 29. $19-28$.

Lartigaudière, C.. Lentheric, I., Puy, J., Pintó, E., 2004. Biochemical characterisation of core browning and brown heart disordets in pear by multivariate analysis. Postharvest Biol. Technol. 31. 29-39.

Marigheto. N.. Duarte, S., Hills, B.P., 2005. An NMR relaxation study of avocado guality. Appl. Magn. Reson. 29, 687-701.

Raffo, A. Gianferti, R., Barbieti, R., Brosio, E., 2005. Ripening of banana fruit monitored by water relaxation and diffusion ${ }^{1} \mathrm{H}-\mathrm{NMR}$ measurements. Food Chem. 89, 149-158.

Saquet, A.A., Streif, J., Bangerth, F. 2003. Energy metabolism and membrane lipid alterations in relation to brown heart development in 'Conference" pears during delayed controlled atmosphere storage. Postharvest Biol. Technol. 30. $123-132$.

Stejskal. E.O., Tanner. J.E., 1965. Spin diffusion measurements: spin echoes in the presence of a time-dependent field gradient. J. Chem. Phys. 42, 288292.

Veltman. R.H., Kho, R.M., van Schaik, A.C.R., Sanders. M.G., Oosterhaven. J., 2000. Ascotbic acid and tissue browning in pears ( $P$ yrus commutis L. cvs. Rocha and Conference) under controlled atmosphete conditions. Postharvest. Biol. Technol. 19, 129-137.

Veltman. R.H.. Lenthéric, I., Van der Plas, L.H.W., Peppelenbos, H.W, 2003. Internal browning in pear fruit (Pyrus communis L. cv Conference) may be a result of a limited availability of energy and antioxidants. Postharvest Biol. Technol. 28. 295-302

Wang. C.Y. Wang, P.C. 1989. Nondestructive detection of core breakdown in 'Bartlett' pears with nuclear magnetic tesonance imaging. HortScience 24, 106-109. 